\title{
PET Is Alive and Well
}

$\mathbf{I}_{n}$

n a recent article on DOTmed.com entitled "Is There Life After PET?" Wayne Webster, founder of ProActics Consulting, wrote that "PET is nearing the end of its life cycle and will be replaced for most if not all of its clinical uses within the next few years." PET was a research device until 1999 because there was no reimbursement by Medicare or any other payer worldwide. The short half-lives of ${ }^{11} \mathrm{C},{ }^{18} \mathrm{~F}$, and other PET nuclides did not fit the well-established nuclear medicine business model. The chief obstacle then and now is obtaining approval of PET radiopharmaceuticals from the Food and Drug Administration (FDA) and Medicare. To get PET procedures reimbursed by Medicare, it is necessary to get FDA approval.

The approval of ${ }^{18} \mathrm{~F}-\mathrm{FDG}$ by the FDA was a major breakthrough to the rapid incorporation of PET into nuclear medicine practice, particularly in oncology. In the 1991 Journal of Nuclear Medicine, I wrote "Clinical PET: Its Time Has Come." The high rate of reimbursement led to profitable operations. Nuclear pharmacies were able to transport ${ }^{18} \mathrm{~F}-\mathrm{FDG}$ by automobile to nearby hospitals.

Webster points out that "taking the path of FDA approval for ${ }^{18}$ FDG set a precedent for the future approval of all PET radiopharmaceuticals. From this point forward new PET radiopharmaceuticals would have to go through the FDA process."

I believe that we should continue to develop the local production and approval of PET radiopharmaceuticals within hospitals when the radiopharmaceuticals will be given to patients in millimolar doses to provide diagnostic information, not to achieve a therapeutic effect. Over the decades before the 1970s, radiopharmaceuticals were ex-

COPYRIGHT (C) 2007 by the Society of Nuclear Medicine, Inc.

DOI: 10.2967/jnumed.48.4.495.07

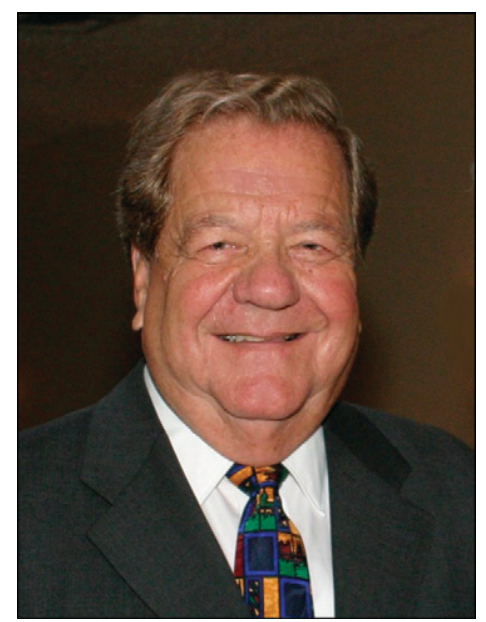

empted from FDA approval. I know of no untoward side effects ever reported from their administration over this long period. Local regulation within hospitals is feasible, operating under guidelines approved by the FDA.

In his clear presentation of the "problems of PET," Webster does not refer to the regulation by the more than 100 Radioactive Drug Research Committees at institutions, chiefly universities or pharmaceutical company research laboratories. In 2003, there were 284 research studies in the United States involving 2,797 human subjects and more than 120 different radioactive molecules. What we need to do now is simplify toxicity studies, as is possible because the PET tracers can label natural body constituents and are administered to patients in micromolar or millimolar quantities, far less than one hundredth of the toxic dose. PET and SPECT tracer studies are particularly helpful in providing surrogate markers for assessing the value of new, stable drugs in the treatment of specific disease states. Surrogate markers can greatly reduce the cost of drug design and development. At present, approval of nonradioactive drugs by the FDA requires clinical trials that show a statistically significant effect on mortality. This requirement requires long- term studies and results in enormous costs. To date, the greatest contribution of molecular imaging is in the faster and cheaper design and discovery of new, effective, stable drugs. Diagnostic radiotracer studies are used initially to characterize the patient ("make the diagnosis") in order to provide homogeneous groups of patients for clinical trials. Treatment will at times be the subsequent administration of larger doses of the same radioactive drug. Pretargeting with nonradioactive molecules given before the diagnostic tracer dose can provide an important way to increase the accumulation of the subsequently administered therapeutic drug. In such cases, the FDA regulations require approval of the pretargeting dose and of the subsequent radioactive tracer drug. Assessing the effects of drugs on the brain will continue to increase the numbers of PET studies in drug design and development. One third of all prescription drugs in the United States are given to affect mental activity. People have taken drugs that affect mental activity since prehistoric times, most often alcohol and opiates.

With Webster, I have long advocated the continual advancement of SPECT/ $\mathrm{CT}$, and I believe it will continue to grow alongside PET/CT. But ${ }^{11} \mathrm{C}$ and ${ }^{18} \mathrm{~F}$ tracers are better suited than SPECT tracers for revealing regional biochemistry. For example, despite great efforts, no one has yet been able to develop a ${ }^{99 \mathrm{~m}} \mathrm{Tc}$ tracer that will reveal glucose utilization. One leading candidate, ${ }^{99 m}$ Tc-glucosamine, was found to behave in a manner similar to labeled thymidine tracers rather than glucose. I do not believe that PET/CT will be replaced with another modality. It will continue to grow, as will SPECT/CT and other partners in molecular imaging.

Henry N. Wagner, Jr. Johns Hopkins Bloomberg School of Public Health

Baltimore, Maryland 